\title{
NGC 5719/13: interacting spirals forming a counter-rotating stellar disc ${ }^{\star}, \star \star$
}

\author{
D. Vergani ${ }^{1,2}$, A. Pizzella ${ }^{3}$, E. M. Corsini ${ }^{3,4}$, W. van Driel $^{2}$, L. M. Buson ${ }^{5}$, R.-J. Dettmar ${ }^{6}$, and F. Bertola ${ }^{3}$ \\ 1 INAF - IASF Milano, via Bassini 15, 20133 Milano, Italy \\ e-mail: daniela@lambrate.inaf.it \\ 2 Observatoire de Paris, Section de Meudon, GEPI, 5 place Jules Janssen, 92195 Meudon Cedex, France \\ 3 Dipartimento di Astronomia, Università di Padova, vicolo dell'Osservatorio 3, 35122 Padova, Italy \\ 4 Scuola Galileiana di Studi Superiori, via VIII Febbraio 2, 35122 Padova, Italy \\ INAF - Osservatorio di Padova, Vicolo dell'Osservatorio 5, 35122 Padova Italy \\ 6 Astronomisches Institut der Ruhr-Universität Bochum, 44780 Bochum, Germany
}

Received 18 September 2006 / Accepted 6 November 2006

\section{ABSTRACT}

\begin{abstract}
Context. When a galaxy acquires material from the outside, it is likely that the resulting angular momentum of the accreted material is decoupled from that of the pre-existing galaxy. The presence of stars counter-rotating with respect to other stars and/or gas represents an extreme case of decoupling.

Aims. NGC 5719, an almost edge-on Sab galaxy with a prominent skewed dust lane, shows a spectacular on-going interaction with its face-on Sbc companion NGC 5713. Observations of such interacting systems provide insight into the processes at work in assembling and reshaping galaxies.

Methods. Studies were made of the distribution and kinematics of neutral hydrogen in the NGC 5719/13 galaxy pair and the ionised gas and stellar kinematics along the major axis of NGC 5719.

Results. Two HI tidal bridges that loop around NGC 5719 and connect to NGC 5713, and two HI tidal tails departing westward from NGC 5713 were detected. There is a correspondence between the HI condensations and the location of clumps of young stars within and outside the disc of NGC 5719. The low-mass satellite PGC 135857 at the tip of the northern tail was detected in HI, and is likely a by-product of the interaction. The neutral and ionised hydrogen in the disc of NGC 5719 are counter-rotating with respect to the main stellar disc. The counter-rotating stellar disc contains about $20 \%$ of the stars in the system, and has the same radial extension as the main stellar disc. This is the first interacting system in which a counter-rotating stellar disc has been detected.

Conclusions. The data support a scenario where HI from the large reservoir available in the galaxy's surroundings was accreted by NGC 5719 onto a retrograde orbit and subsequently fuelled the in situ formation of the counter-rotating stellar disc.
\end{abstract}

Key words. galaxies: individual: NGC 5713 - galaxies: individual: NGC 5719 - galaxies: interactions galaxies: kinematics and dynamics - galaxies: spiral

\section{Introduction}

Strong interactions between galaxies often produce huge tidal tails consisting of both cold gas and stripped, or even newborn stars, as shown by numerical simulations as well as by detailed observations (see Schweizer 2000, for a review). Gas plays a crucial role in such interactions, because of its dissipative nature. When a galaxy acquires material from outside, it is likely that the resulting angular momentum of the acquired material is decoupled from that of the pre-existing galaxy. The presence of stars counter-rotating with respect to other stars and/or gas represents a truly extreme case of decoupling. This phenomenon has been detected in the central regions of a large number of galaxies, with morphological types ranging from ellipticals to S0's and spirals (see Bertola \& Corsini 1999, for a review). Although it is usually invoked as the signature of a past external event, some attempts have been made to explain special cases of stellar counter-rotating discs as due to a self-induced

\footnotetext{
* Based on observations carried out at the European Southern Observatory (ESO 69.B-0515).

$\star \star$ Figure 4 is only available in electronic form at http://www . aanda.org
}

phenomenon in non-axisymmetric potentials (Evans \& Collett 1994; Athanassoula et al. 1996; Wozniak \& Pfenninger 1997).

Extended stellar counter-rotation in discs of S0's and spirals appears to be a rare phenomenon (Kuijken et al. 1996; Kannappan \& Fabricant 2001; Falcón-Barroso et al. 2006). It has been argued that the retrograde acquisition of small amounts of external gas can give rise to counter-rotating gaseous discs in gas-poor S0's only, while in gas-rich spirals the newly acquired gas is swept away by the pre-existing gas. Counter-rotating gaseous and stellar discs in spirals are formed only from, respectively, the retrograde acquisition of amounts of gas larger than the pre-existing gas content, and subsequent star formation (Pizzella et al. 2004).

To date only few cases of extended counter-rotating stellar discs have been observed. The S0 galaxy NGC 4550 has two cospatial counter-rotating stellar discs, one of which is co-rotating with the gaseous one (Rubin et al. 1992). The two discs have exponential surface brightness profiles with the same central surface brightness and scale lengths (Rix et al. 1992), but different thickness (Emsellem et al. 2004). In the early-type spirals NGC 4138 (Jore et al. 1996) and NGC 7217 (Merrifield \& Kuijken 1994) about $20-30 \%$ of the disc stars reside in 
retrograde orbits. These counter-rotating stellar discs have the same spatial extent but a lower surface brightness than the main stellar disc. In NGC 7217 the gaseous disc rotates in the same direction as the main stellar disc. The contrary is true for NGC 4138, whose counter-rotating disc is currently forming stars. The Sa galaxy NGC 3593 (Bertola et al. 1996) is composed of a radially more concentrated stellar disc that contains about $20 \%$ of the total luminous mass and dominates the stellar kinematics in the inner kpc, and a counter-rotating stellar disc that is radially more extended and dominates the outer kinematics. The two discs have exponential luminosity profiles with different scale lengths and central surface brightnesses. The gaseous disc co-rotates with the more concentrated disc, which is characterized by a high star formation rate (Corsini et al. 1998; García-Burillo et al. 2000).

The NGC 5719/13 pair is the subject of the present paper. NGC 5719 is a highly inclined Sab galaxy system with a complex, asymmetric morphology. It is characterized by a prominent dust lane skewed by about $25^{\circ}$ with respect to the optical main body, and a chain of faint condensations in the north-western section of the outermost disc. Although it has been classified as a barred galaxy by de Vaucouleurs et al. (1991, hereafter RC3), surface photometry shows no clear sign of a bar (Vergani et al. 2006). Its face-on Sbc companion NGC 5713 has an overall undisturbed look in the optical, but it shows a conspicuous barlike distortion pointing toward NGC 5719 in the near-infrared (Mulchaey et al. 1997).

The pair belongs to the rich LGC 386 group (Garcia 1993). The projected separation between the two galaxies is $77 \mathrm{kpc}(11.5)$ and their systemic velocities differ by about $160 \mathrm{~km} \mathrm{~s}^{-1}$. Although their relative proximity and peculiar morphologies already suggested they are interacting (de Vaucouleurs \& de Vaucouleurs 1964), it is only in the HI line images (Sect. 3.1) that we can really appreciate the amount and extent of the displaced and accreted material. Some basic properties of the two galaxies are given in Table 1.

In this paper we report the detection of a counter-rotating stellar disc in the spiral galaxy NGC 5719 which is interacting with its close companion galaxy NGC 5713. The pair offers a unique opportunity to study the effects of an on-going acquisition event in the framework of the origin of counter-rotating stellar discs. The paper is organized as follows. The optical and radio observations and data reduction are described in Sect. 2. The results are presented in Sect. 3 and discussed in Sect. 4, and our conclusions are given in Sect. 5 .

\section{Observations and data reduction}

\subsection{Radio observations}

We observed the NGC 5719/13 pair with the Very Large Array (VLA) radio synthesis telescope in its $\mathrm{C}$ configuration in April 2000 as part of a study of the kinematics of galaxies with box/peanut shaped bulges (Vergani et al. 2006). The galaxies were observed for $8 \mathrm{~h}$ in the 4ABCD spectral line mode with two partially overlapping spectral bands, each with 64 channels of $24.4 \mathrm{kHz}$ width and a total bandwidth of $2.685 \mathrm{MHz}$. The baselines range between $35 \mathrm{~m}$ and $3400 \mathrm{~m}$. The data cube used for further analysis has 109 velocity channels with a velocity resolution of $5.15 \mathrm{~km} \mathrm{~s}^{-1}$ that cover a velocity range of $\sim 562 \mathrm{~km} \mathrm{~s}^{-1}$, an $H P B W$ of $15^{\prime \prime} .5 \times 14^{\prime \prime}$. 1, and an rms noise level per channel of $0.53 \mathrm{mJy}^{\text {beam }}{ }^{-1}$.

A contour map of the HI column density distribution smoothed to a resolution of $30^{\prime \prime} \times 30^{\prime \prime}$ is shown superimposed
Table 1. Basic parameters of the NGC 5719/13 galaxy pair.

\begin{tabular}{lll}
\hline \hline & NGC 5719 & NGC 5713 \\
\hline$\alpha(\mathrm{J} 2000)$ & $14^{\mathrm{h}} 40^{\mathrm{m}} 56^{\mathrm{s}} .8$ & $14^{\mathrm{h}} 40^{\mathrm{m}} 11^{\mathrm{s}} .6$ \\
$\delta(\mathrm{J} 2000)$ & $-00^{\circ} 19^{\prime} 04^{\prime \prime}$ & $-00^{\circ} 17^{\prime} 26$ \\
Type & SABab(s) pec & SABbc(rs) pec \\
$D_{25}(\mathrm{kpc})$ & 21.8 & 20.2 \\
$i\left(^{\circ}\right)$ & 70 & 27 \\
$\mathrm{PA}\left({ }^{\circ}\right)$ & 96 & 12 \\
$V_{\text {sys }}\left(\mathrm{km} \mathrm{s}^{-1}\right)$ & $1741 \pm 8$ & $1899 \pm 8$ \\
$M_{B, T}^{0}$ & -19.40 & -20.43 \\
\hline
\end{tabular}

NOTES - The centre position, morphological type and optical diameter at a surface brightness level of $25 B$-mag $\operatorname{arcsec}^{-2}$ are taken from the RC3. The inclination is derived as $\cos ^{2} i=\left(q^{2}-q_{0}^{2}\right) /\left(1-q_{0}^{2}\right)$. The observed axial ratio $q$ is taken from the RC3 and an intrinsic flattening of $q_{0}=0.11$ (Guthrie 1992) was assumed. The position angle of optical major axis is taken from HyperLeda. The heliocentric velocity derived from HI data is from Vergani et al. (2006). The absolute total blue magnitude is corrected for inclination and extinction and is taken from the RC3.

on an optical image from the Digitized Sky Survey in Fig. 1. The velocity field (Fig. 1) was obtained by the modified envelopetracing method, which describes the kinematics in edge-on galaxies better than a simple first moment analysis. We refer to Vergani et al. (2006) for further details on the data reduction and velocity field determination.

\subsection{Optical spectroscopy}

We took optical long-slit spectra of NGC 5719 at the European Southern Observatory (ESO) in La Silla with the New Technology Telescope (NTT) on July, 2002. The ESO MultiMode Instrument (EMMI) was used in the Red Medium Dispersion Spectroscopy (REMD) configuration. Grating No. 6 with 1200 grooves $\mathrm{mm}^{-1}$ blazed at $5500 \AA$ was used in the first order in combination with a $1^{\prime \prime} .0$ - wide slit and the mosaiced MIT/LL CCDs No. 62 and 63 with $2048 \times 4096$ pixels of $15 \times$ $15 \mu \mathrm{m}$ each. The wavelength range between 4800 and $5500 \AA$ was chosen to record simultaneously the $\mathrm{H} \beta$ and [OIII] $\lambda 5007 \AA$ emission lines and the MgI absorption triplet at $\sim 5170 \AA$. The instrumental resolution, obtained by measuring the width of sky lines on a science frame after the wavelength calibration, was $F W H M=1.16 \AA\left(\sigma \approx 30 \mathrm{~km} \mathrm{~s}^{-1}\right)$ at $5100 \AA$. The on-chip $2 \times 2$ pixel binning provided a reciprocal dispersion of $0.4 \AA$ pixel $^{-1}$ and an angular sampling of 0.33 pixel $^{-1}$.

The slit was centred on the galaxy nucleus using the guiding TV camera and aligned along the optical major axis $\left(\mathrm{PA}=96^{\circ}\right)$. Two spectra of 50 min each were obtained. A comparison lamp exposure was obtained after each object integration to allow accurate wavelength calibration. Quartz lamp and twilight sky flatfields were used to remove pixel-to-pixel variations and largescale illumination patterns. Several $G$ and $K$ stars were observed with the same set-up to serve as templates in measuring the stellar kinematics. The two spectra were bias subtracted, flat-field corrected, cleaned from cosmic rays, wavelength calibrated, coadded and sky subtracted using standard MIDAS ${ }^{1}$ routines. The stellar kinematics was measured from the galaxy absorption features present in the wavelength range. We used the Fourier Correlation Quotient method (FCQ, Bender 1990) following the

1 MIDAS is developed and maintained by the European Southern Observatory. 

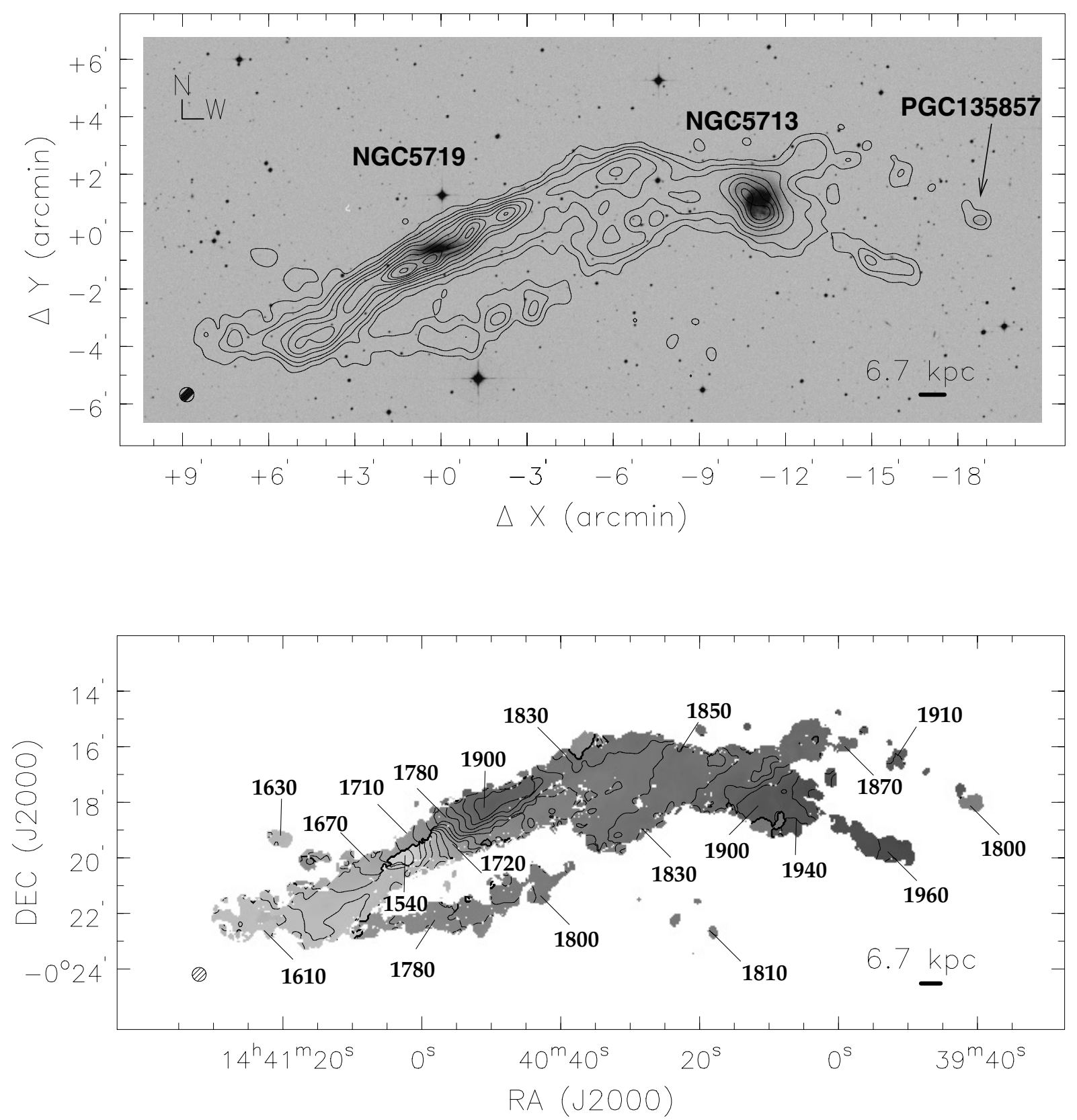

Fig. 1. (Top) Contour map of the HI column density distribution of NGC 5719/5713 superimposed on an optical (DSS2) image. The lowest contour level is at $7.0 \times 10^{19}$ atoms cm${ }^{-2}$, and the increment is $2.4 \times 10^{20}$ atoms cm ${ }^{-2}$. The spatial resolution is $30^{\prime \prime} \times 30^{\prime \prime} .1^{\prime}$ is $\sim 6.7 \mathrm{kpc}$ at the assumed distance of $23.2 \mathrm{Mpc}$. (Bottom) HI velocity field with contours and grey scales from $1500 \mathrm{~km} \mathrm{~s}^{-1}$ (approaching: eastern side, light shading) to $2000 \mathrm{~km} \mathrm{~s}^{-1}$ (receding: western side, dark shading), in increments of $25 \mathrm{~km} \mathrm{~s}^{-1}$.

prescriptions of Bender et al. (1994). The spectra were binned along the spatial direction to obtain a nearly constant signalto-noise ratio larger than 20 per resolution element. The galaxy continuum was removed row-by-row from each spectrum by fitting a fourth to sixth order polynomial. The template spectrum used was that of the K0.5 III star HR5196. The line-of-sight stellar velocity $\left(v_{\star}\right)$, and velocity dispersion $\left(\sigma_{\star}\right)$ were determined by fitting a Gaussian to the line-of-sight velocity distribution (LOSVD) at each radius. Velocities were corrected to heliocentric. We derived errors on the stellar kinematics from photon statistics and CCD read-out noise, calibrating them by Monte Carlo simulations as done by Gerhard et al. (1998). These errors do not take into account possible systematic effects due to template mismatch.
The ionised gas kinematics was measured by simultaneous Gaussian fitting of the $\mathrm{H} \beta$ and [OIII] $\lambda 5007 \AA$ emission lines in the resulting spectrum. The galaxy continuum was removed from the spectra, as was done for measuring the stellar kinematics. We fitted in each row of the continuum-subtracted spectrum a Gaussian to each emission line, assuming them to have the same line-of-sight velocity $\left(v_{\mathrm{g}}\right)$ and velocity dispersion $\left(\sigma_{\mathrm{g}}\right)$. Velocities and velocity dispersions were corrected to heliocentric and for instrumental velocity dispersion, respectively. Far from the galaxy centre (for $|r|>20^{\prime \prime}$ ) we averaged adjacent spectral rows in order to increase the signal-to-noise ratio of the emission lines. The uncertainties in the kinematic parameter determination were derived from photon statistics and CCD read-out noise by means of Monte Carlo simulations. 


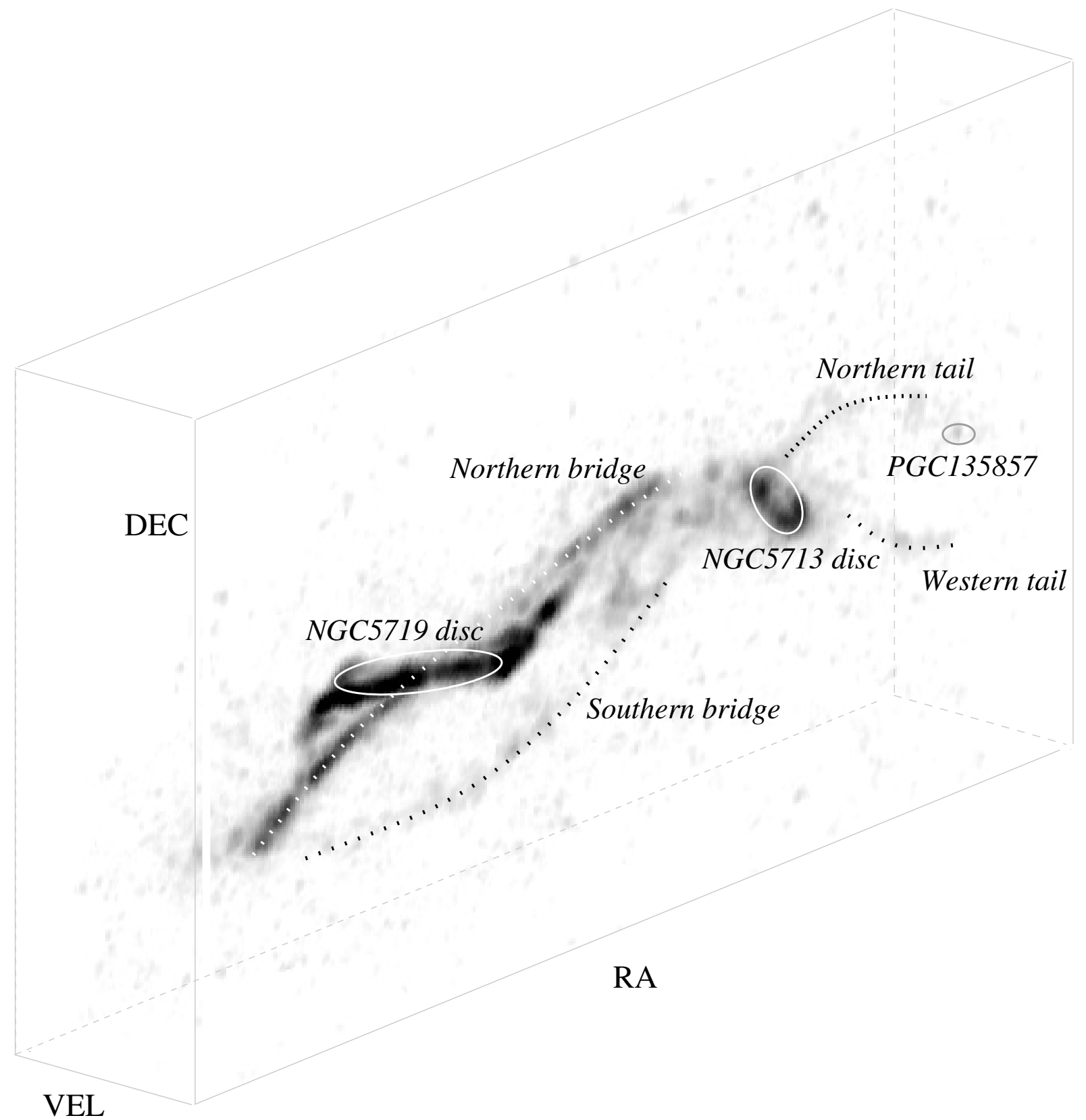

Fig. 2. A three-dimensional representation of the HI emission in the NGC 5719/13 galaxy pair with a different viewing angle than Fig. 1. Here the cube is rotated by $20^{\circ}$ around the declination-axis and by $50^{\circ}$ around the velocity-axis to better visualise the different structures (marked in the figure).

\section{Results}

In this section we describe the distribution and kinematics of the stellar and the cold+ionised gaseous components of the NGC 5719/5713 system, in particular the on-going merging event in the galaxy pair and the detection of two counter-rotating stellar discs in NGC 5719.

\subsection{HI morphology and kinematics}

The most striking features of the HI morphology are due to the on-going interaction between NGC 5719 and NGC 5713, which is mostly unnoticeable at optical wavelengths (Fig. 1).

In NGC 5719 the HI extends to over five times its optical size on both sides, reaching a radius of $8^{\prime}$ or $54 \mathrm{kpc}$ at the assumed distance of $23.2 \mathrm{Mpc}$, compared to the $180 \mathrm{kpc}$ overall HI extent of the pair. The major axis of its HI distribution follows that of the oblique dust lane $\left(\mathrm{PA}=121^{\circ}\right)$, and both are skewed by $25^{\circ}$ to that of the optical body $\left(\mathrm{PA}=96^{\circ}\right)$. The gas associated with its disc is regularly distributed within the optical boundaries and bends away beyond $\sim 1$ ' 1 , forming an integral-shaped warp. The NW side is receding (Fig. 1).

In its NW outermost optical disc the HI distribution peaks where a chain of faint optical condensations is seen. In particular, the clumps number 1 and 2 in Neff et al. (2005) coincide with $\mathrm{HI}$ condensations at radial velocities of $\sim 1637 \mathrm{~km} \mathrm{~s}^{-1}$ and $\sim 1529 \mathrm{~km} \mathrm{~s}^{-1}$, respectively.

Its HI kinematics are globally quite chaotic, especially outside the optical body, as commonly found in on-going mergers.

Inspection of the 3D data cube (Fig. 2) shows that all HI structures, which are seen in projection in the HI maps, 

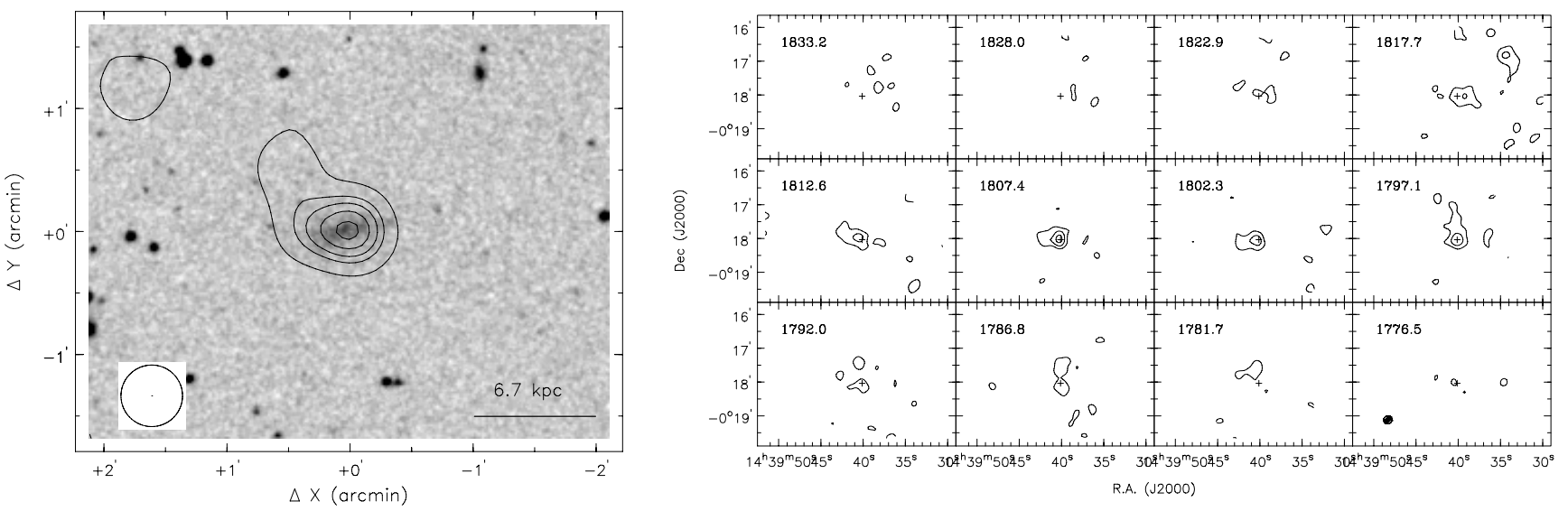

Fig. 3. (Left) Contour map of the HI column density distribution of the faint companion PGC 135857 superimposed on an optical (DSS2) image. The lowest contour level is at $6 \times 10^{19}$ atoms cm $\mathrm{cm}^{-2}$ and the increment is $9 \times 10^{19}$ atoms $\mathrm{cm}^{-2}$. (Right) Channel maps of the companion galaxy PGC 135857. Contour levels are at $-1,1(\sim 2 \sigma)$ to $3 \mathrm{mJy} \mathrm{beam}^{-1}$, in steps of $1 \mathrm{mJy}^{-1} \mathrm{beam}^{-1}$. The spatial resolution is $30^{\prime \prime} \times 30^{\prime \prime}$. The cross represents the optical centre of PGC 135857.

actually reside in different planes. As a consequence of projection effects the velocity map shows sharp discontinuities at, e.g., $1780 \mathrm{~km} \mathrm{~s}^{-1}$ and $1560 \mathrm{~km} \mathrm{~s}^{-1}$ (Fig. 1, lower panel).

From the nearly face-on HI disc in NGC 5713 a bridge points toward and connects to the NW tip of the HI disc in NGC 5719, which is marked as Northern bridge in Fig. 2. It extends over $114 \mathrm{kpc}$ on the sky and has a large velocity gradient of $300 \mathrm{~km} \mathrm{~s}^{-1}$. South of the main HI disc in NGC 5719 lies a weaker and clumpy bridge, marked as Southern bridge in Fig. 2, which starts from the NW tip of the NGC 5719 disc at $\sim 1650 \mathrm{~km} \mathrm{~s}^{-1}$, loops around NGC 5719 and moves towards NGC 5713 with receding velocities, assuming that the structure is trailing.

We also detected HI emission from PGC 135857, a small $B_{t}=17.40 \mathrm{Sm}$-type satellite with $v_{\text {sys }}=1802 \mathrm{~km} \mathrm{~s}^{-1}, \sim 63.6 \mathrm{kpc}$ E of the centre of NGC 5713 (see Fig. 3). Although our observations are affected by primary-beam attenuation $\left(F W H P=30^{\prime}\right)$, we detected a fair amount of $\mathrm{HI}$ in it $\left(M_{\mathrm{HI}} / L_{B} \sim 0.18 M_{\odot} / L_{\odot, B}\right)$. In its close surroundings we detected two low column density HI tails at $v \sim 1800-1950 \mathrm{~km} \mathrm{~s}^{-1}$ : the Northern and Western tails, see Fig. 2. The Northern tail is likely physically connected to PGC 135857, as shown by the channel maps Fig. 4.

NGC 5719 has a total $\mathrm{HI}$ mass $M_{\mathrm{HI}}$ of $7.2 \times 10^{9} M_{\odot}$ and a mean HI surface density of $5.11 M_{\odot} \mathrm{pc}^{-2}$ - values which lie in the lower quartile of the distributions determined for $\mathrm{Sab} / \mathrm{Sb}$ galaxies, although its $M_{\mathrm{HI}} / L_{B}$ ratio of $0.98 M_{\odot} / L_{\odot}, B$ is significantly higher than the median value found by Roberts \& Haynes (1994). NGC 5713 has $M_{\mathrm{HI}}=6.6 \times 10^{9} M_{\odot}$, which is at the lower limit of the average value for $\mathrm{Sb} / \mathrm{Sbc}$ galaxies (Roberts \& Haynes 1994). For the tidal features we estimated an $M_{\mathrm{HI}}$ of $2.3 \times 10^{8} M_{\odot}$, i.e. about $15 \%$ of that of the NGC $5719 / 13$ system, by visually selecting these features in each channel map where they could be distinguished from the main emission. This value is likely to be a lower limit due to projection effects.

In Fig. 5 we present the HI position-velocity diagrams (PVDs) along the HI major axes of NGC $5719\left(\mathrm{PA}=121^{\circ}\right)$ and NGC $5713\left(\mathrm{PA}=11.5^{\circ}\right)$. In both PVDs the kinematics are fairly chaotic, indicating the signatures of several planes of HI emission. Figure 6 shows the PVD along the optical major axis of NGC $5719\left(\mathrm{PA}=96^{\circ}\right)$, as well as a zoomed-out version that shows the $74 \mathrm{kpc}$ long tail connecting NGC 5719 and NGC 5713. The maximum rotation velocities derived for the ionised gas, and the co- and counter-rotating stars (see next section) are shown for comparison. It is clear that only a small fraction of the HI in NGC 5719 resides along the optical major axis and that most of it lies along $\mathrm{PA}=121^{\circ}$.

\subsection{Stellar and ionized gas kinematics}

The kinematics of the ionised gas and stars in NGC 5719 are very complex. There are three distinct kinematical components. The rotation of the main stellar component, which represents $\sim 80 \%$ of the galaxy's light, is receding towards the SE quadrant (Fig. 7) - we have assumed this as the reference sense of rotation of the galaxy. The ionised and HI gas are rotating in the opposite sense. The presence of a second, counter-rotating stellar component is revealed by the double-peaked stellar LOSVD (Fig. 8).

At $|r|>10^{\prime \prime}$ the main and counter-rotating components can be disentangled due to their large difference in line-of-sight velocities $\left(\Delta v_{\star} \sim 350 \mathrm{~km} \mathrm{~s}^{-1}\right)$ and small velocity dispersions $\left(\sigma_{\star}<100 \mathrm{~km} \mathrm{~s}^{-1}\right)$. The X-shaped pattern is the signature of counter-rotation (see for comparison Fig. 11.18 of Binney \& Merrifield 1998). At $|r| \sim 10^{\prime \prime} \Delta v_{\star}$ decreases and the two components blend with a large LOSVD $\left(\sigma_{\star} \sim 240 \mathrm{~km} \mathrm{~s}^{-1}\right)$, which is likely due to the combined $\Delta v_{\star}$ of the two unresolved components rather than to an increase of their intrinsic velocity dispersion. In this region we measured $v_{\star} \sim 0 \mathrm{~km} \mathrm{~s}^{-1}$, indicating that the two components have roughly the same intensity. At small radii the main component dominates the observed kinematics. The features observed in the radial profile of $\sigma_{\star}$ are also indicative of the presence of a counter-rotating component. In the region where the two components are unblended they both show low values of $\sigma_{\star}$, which we expect for a rotating stellar disc $\left(\sigma_{\star} \sim 80 \mathrm{~km} \mathrm{~s}^{-1}\right)$. At $|r|<5^{\prime \prime} \sigma_{\star}$ is constant, suggesting that only one component is present in this region.

We derived the relative intensity of the counter-rotating component as a function of radius. We constructed a simple kinematical model by using the spectrum of the stellar template to build the galaxy spectrum. We adopted for the prograde and retrograde components the values of the line-of-sight velocity and velocity dispersion we fitted to the stellar and ionised-gas kinematics, respectively.

For each radius a synthetic galaxy spectrum was obtained by co-adding the two spectra we derived by convolving the stellar template spectra of the two counter-rotating components. The relative intensities of the two components were constrained by 

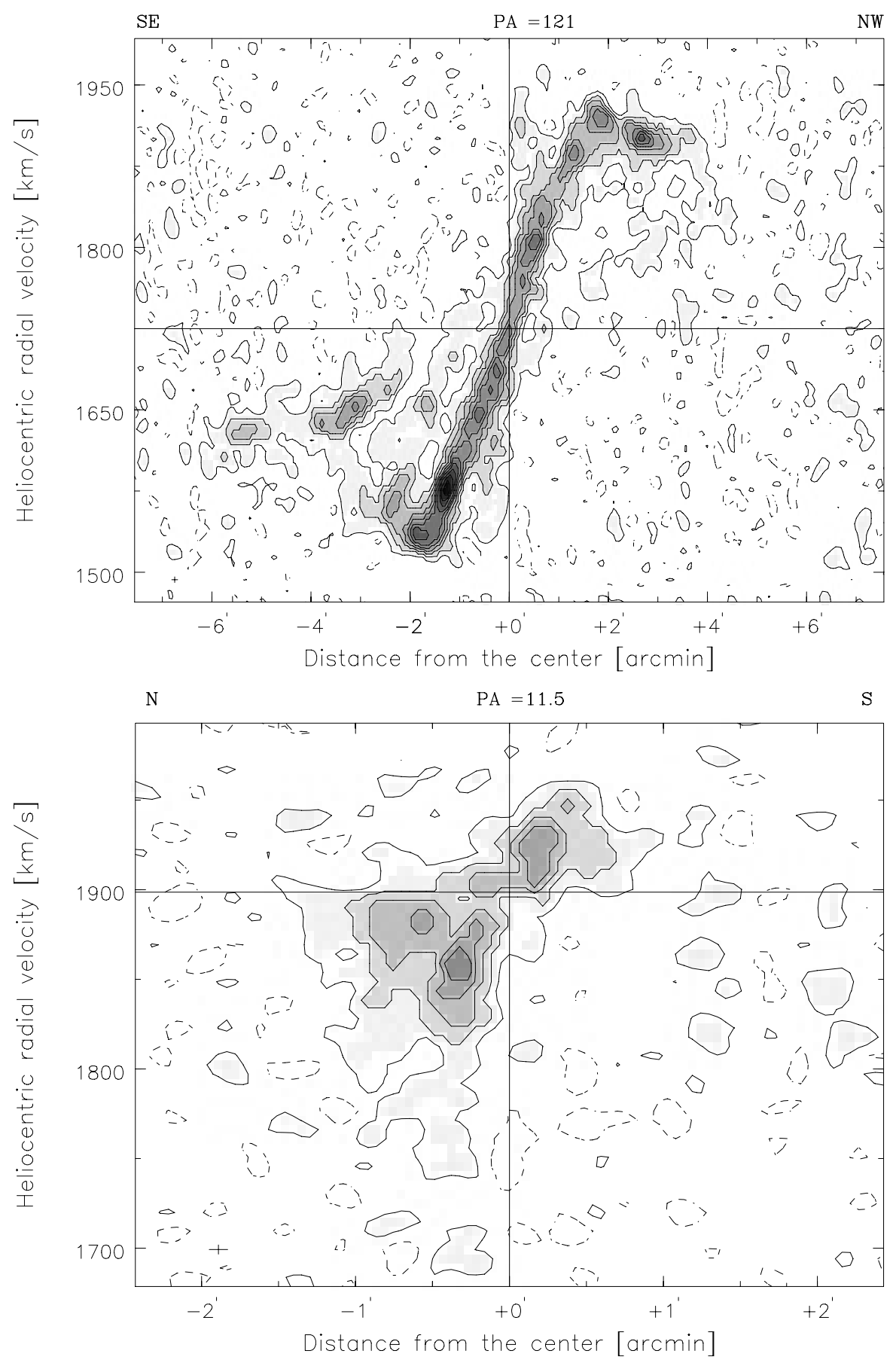

Fig. 5. Position-velocity diagrams along the HI major axis of NGC $5719\left(\mathrm{PA}=121^{\circ}\right.$, top $)$ and $\mathrm{NGC} 5713\left(\mathrm{PA}=11.5^{\circ}\right.$, bottom $)$. The horizontal lines indicate the systemic velocities of the galaxies. Contour levels are at $-3,-1.5$ (dashed), and from 1.5 to $27 \sigma$, in steps of $1.5 \sigma(1 \sigma \approx$ $0.5 \mathrm{mJy}$ beam $^{-1}$ ). The angular and velocity resolutions are $15^{\prime \prime}$ and $5.4 \mathrm{~km} \mathrm{~s}^{-1}$, respectively (crosses at the bottom left side).

comparison of the $v_{\star}$ and $\sigma_{*}$ values measured in the synthetic and observed spectra. We found that for $|r|<3^{\prime \prime}$ the data are consistent with the absence of counter-rotating stars, although we can not exclude them contributing up to $30 \%$ of the total light. At $|r| \sim 8^{\prime \prime}$ the counter-rotating component reaches its maximum intensity ( $\sim 50 \%$ of the total light). At larger radii its contribution decreases to $\sim 30 \%$ on the SE side and $\sim 20 \%$ on the NW side, respectively.

Our observations are consistent with the measurements of the ionised-gas and stellar kinematics by Rhee et al. (2004). Due to a lower spectral resolution they measured no rotation in the very centre of NGC 5719, but did not resolve the counter-rotating component. Nevertheless, at large radii on the SE side their data show the presence of stars rotating at $v_{\star} \lesssim 100 \mathrm{~km} \mathrm{~s}^{-1}$ in an opposite direction than the ionised gas and corresponding to the most luminous stellar component of NGC 5719.

\section{Discussion}

Both NGC 5719 and NGC 5713 are characterized by morphological and kinematical peculiarities. NGC 5719 has a skewed dust lane and shows faint condensations at its optical edge, and NGC 5713 appears very lopsided. The on-going interaction between the galaxies is evident from the HI distribution and 


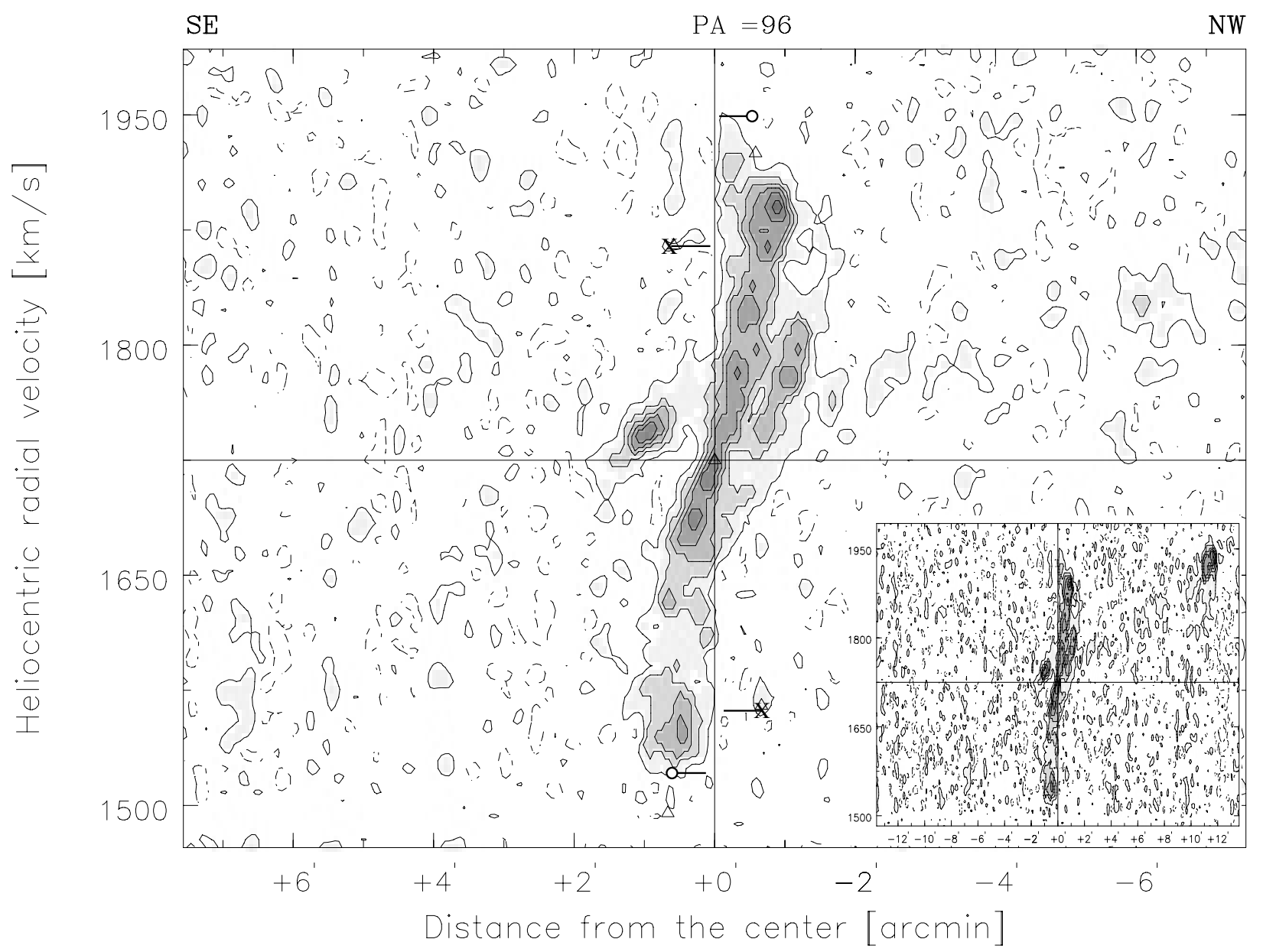

Fig. 6. HI position-velocity diagram obtained along the $\mathrm{PA}=96^{\circ}$ optical major axis of NGC 5719, with overlaid the rotation curves of the main stellar disc (crosses), the ionised gas (circles), and counter-rotating stars (triangles). (Main panel): the horizontal line indicates the systemic velocity of NGC 5719. The contour levels are at $-3,-1.5$ (dashed), and from 1.5 to $27 \sigma$ in steps of $1.5 \sigma\left(1 \sigma \approx 0.5 \mathrm{mJy}_{\text {beam }}^{-1}\right)$. The angular and velocity resolutions are $15^{\prime \prime}$ and $5.4 \mathrm{~km} \mathrm{~s}^{-1}$, respectively. (Inset panel:) HI position-velocity diagram $\left(\mathrm{PA}=96^{\circ}\right.$ ) with a larger radial coverage to show the emission from NGC 5713 at $+11^{\prime}$ from the centre.

kinematics, which show an intricate system extending over more than $27^{\prime}$ in different planes in the sky. This indicates that gas was removed from NGC 5713 by NGC 5719 and/or stripped from their discs into the vicinity, probably during different close passages. The kinematics of both HI discs are disturbed, particularly in the portions which are close to the bridges that connect the two galaxies. We speculate that a substantial fraction (at least $10 \%$ ) of the entire HI mass of the system that now resides in the tidal features around NGC 5719, may once have belonged to NGC 5713, which is presently relatively gas-poor for its morphological classification. The presence of the small satellite galaxy PGC 135857 seems to be related to the interaction of the main galaxy pair, and it might have formed as a result of a large local HI reservoir being subjected to the physical conditions that triggered star formation.

NGC 5719 hosts a counter-rotating component which contains both ionised and neutral hydrogen, as well as $\sim 20 \%$ of the disc stars. The counter-rotating stellar disc has the same spatial extent as the main stellar disc component. Compared to the other known cases of spiral galaxies with counter-rotating stellar discs, the counter-rotating stars in NGC 5719 have the same radial distribution as in NGC 4138 (Jore et al. 1996) and NGC 7217 (Merrifield \& Kuijken 1994), and they rotate in the same sense as the gaseous component, like in NGC 4138 and NGC 3593 (Bertola et al. 1996). All these counter-rotating discs have the same fraction (20-30\%) of the total luminosity of the host galaxy. On the other hand, NGC 5719 is the only known object belonging to an interacting system with stripping and/or gas accretion gas that has stellar counter-rotating discs. This give us a unique opportunity to shed light on the origin of a counterrotating stellar disc, as the end result of the star formation occurred in newly acquired material due to galaxy interaction.

The ionised gas rotates in the same direction and in the same plane as the HI disc. They both counter-rotate with respect to the main stellar disc and have nearly identical velocity amplitudes $\left(\Delta v \approx 440 \mathrm{~km} \mathrm{~s}^{-1}\right)$. The velocity amplitude of the counterrotating stars is close to that of the neutral and ionised gas, while that of the main stellar disc is lower $\left(\Delta v_{\star} \lesssim 320 \mathrm{~km} \mathrm{~s}^{-1}\right)$.

Given its kinematical properties, it is reasonable to invoke a galaxy interaction as the origin of the stellar counter-rotation in the NGC 5719 disc, rather than internal mechanisms. The radial velocity amplitudes of the various components indicate that the counter-rotating gaseous disc and the subsequent in situ stellar formation that occurred in it are attributable to the acquired HI.

As in NGC 4138 (Jore et al. 1996) the data suggest that the most of the stars in the counter-rotating disc formed later from the ionised-gas disc, as commonly found in normal galaxies.

Population synthesis analysis has been used (e.g., Carollo et al. 1997) to determine the age difference between the co-rotating and counter-rotating stellar components in 


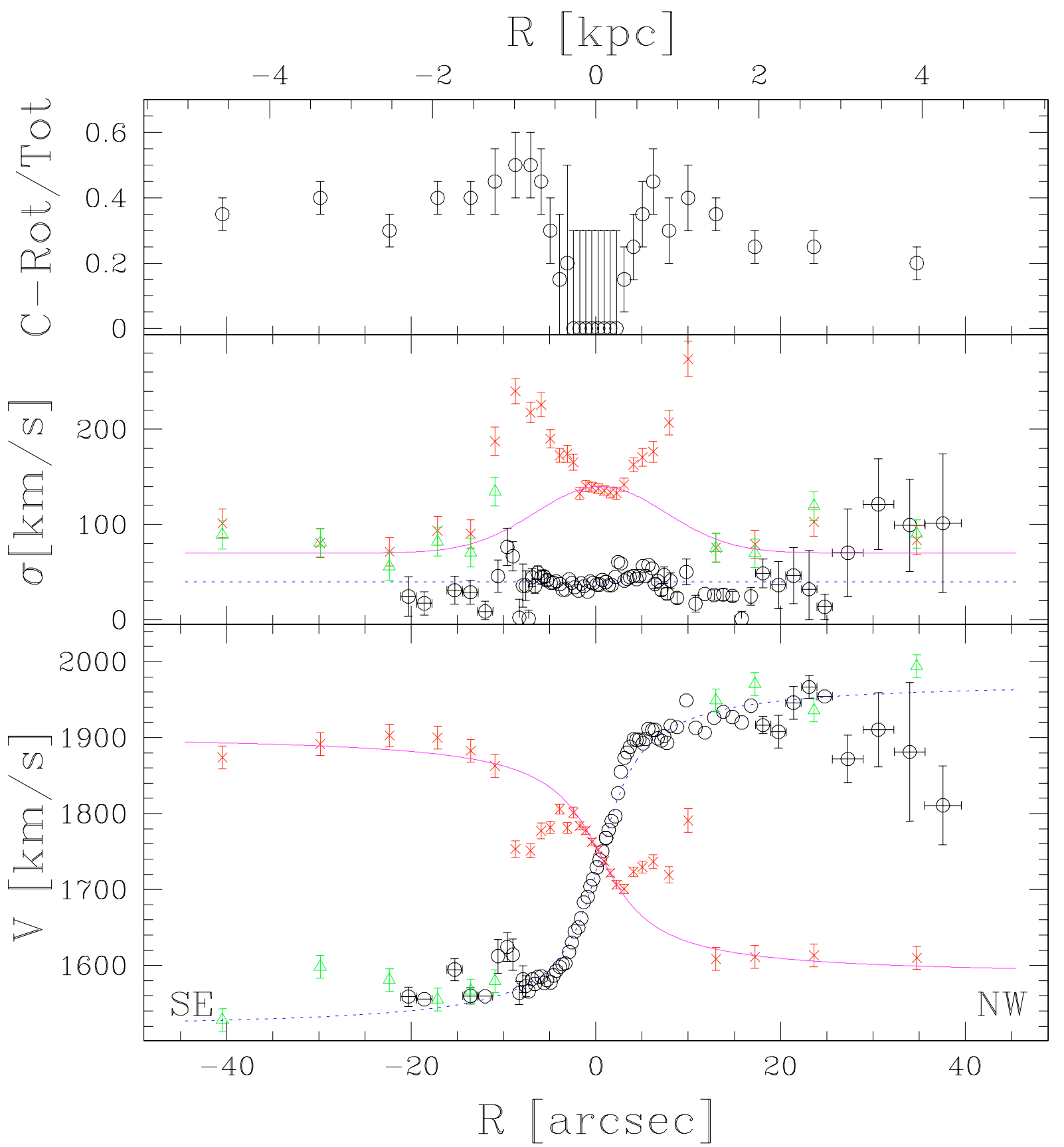

Fig. 7. (Bottom panel) Heliocentric radial velocities as function of position along the optical major axis $\left(\mathrm{PA}=96^{\circ}\right)$ of the ionised gas $($ circles $)$, the main stellar component (red crosses) and the counter-rotating stars (green triangles). (Middle panel) The velocity dispersion of the different components: co- and counter-rotating stellar populations, and the gaseous component (see details in the bottom panel). (Top panel) The luminosity fraction of counter-rotating stars as function of radius in NGC 5719; see the text for details.

kinematically-decoupled cores in ellipticals. Recent results (Morelli et al. 2004; McDermid et al. 2006) show that most of these cores, which are at most a few hundred parsec in size, are younger than the rest of the host galaxy. This favours the in situ stellar formation scenario in galactic cores. However, this technique has never been applied to large-scale $(>1 \mathrm{kpc})$ counterrotating discs, which are subject to different physical conditions and processes than galactic cores. The spectroscopic resolution of our data does not allow this kind of analysis. Moreover, the counter-rotating component of NGC 5719 does not dominate the light distribution at any radius, making measurements of its line strength indices infeasible. We therefore adopted other diagnostics to investigate the origin of stars in the counter-rotating disc.
Neff et al. (2005), combining the GALEX UV data with VLA HI data obtained using a configuration similar to ours (presented succinctly in Langston \& Teuben 2001), already pointed out that there is a strict coincidence between the location of the UV-bright regions and HI column density peaks within and far from the NGC 5719 disc. The stars dominating the UV light in the NGC 5719 disc are very young (300-400 Myr), whereas other regions outside the main stellar disc appear to be even younger (2-200 Myr), and there are many locations in the northern and southern bridges where bright UV clumps were detected. The young age of the stellar population is consistent with the dynamical age of the HI bridges (e.g., Mihos \& Hernquist 1994, 1996), which probably exceeds $500 \mathrm{Myr}$, the estimated epoch of 


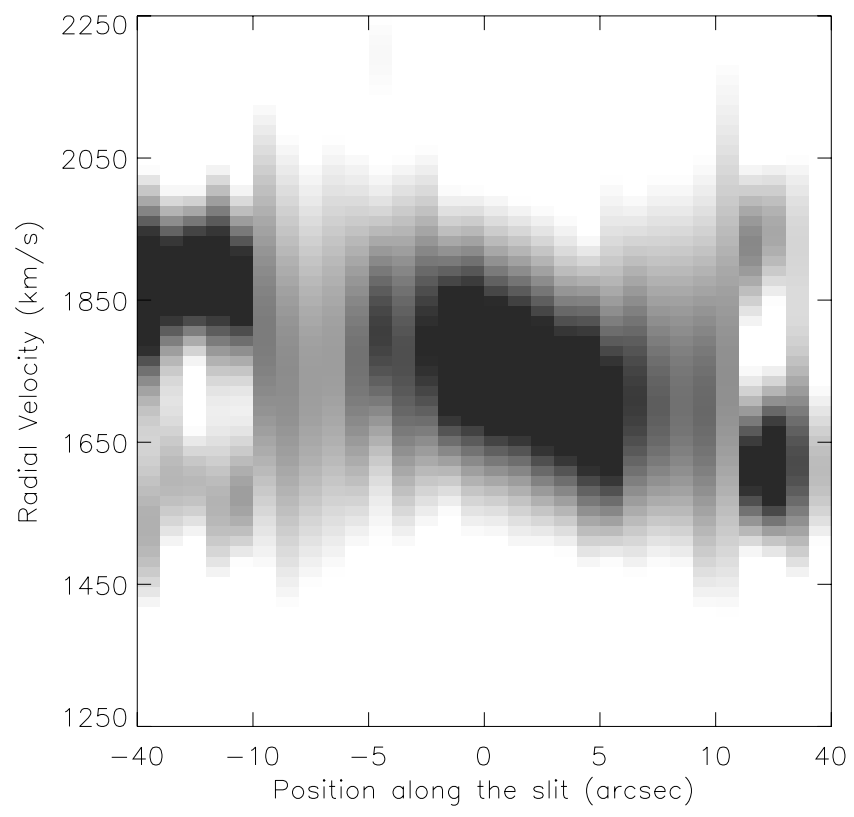

Fig. 8. Grey-scale plot of the stellar line-of-sight velocity distribution (LOSVD) in NGC 5719, showing the projected stellar intensity along the optical $\left(\mathrm{PA}=96^{\circ}\right)$ major axis, where the greyscale represents the stellar intensity, from zero (white) to maximum (black). The two counter-rotating stellar discs are clearly identifiable at $r \geq 10^{\prime \prime}$ through the characteristic $\mathrm{X}$-shaped pattern (see text). For presentation purposes the spatial scale is not linear.

the closest approach between the two galaxies. This epoch was derived from their current projected separation $(\sim 80 \mathrm{kpc})$ and assumed relative velocity $\left(\sim 200 \mathrm{~km} \mathrm{~s}^{-1}\right)$. Although we can not directly associate the young UV condensations with the stars in the counter-rotating disc due to the 5-6" angular resolution of GALEX (Morrissey et al. 2005) and projection effects, the spatial HI-UV association in the NGC 5719 disc, bridges, and tails indicate that the accreted gas in the counter-rotating disc is actually forming a substantial number of stars. All these data support a scenario where the neutral hydrogen from the large reservoir available in the surroundings was first accreted by NGC 5719 onto a retrograde orbit and subsequently formed in situ into a counter-rotating stellar disc.

We argue that PGC 135857 was formed in the interaction process, as suggested by the frequency with which such dwarf companions are observed in similar cases like Stephan's Quintet (Mendes de Oliveira et al. 2004), the Antennae (Hibbard et al. 2005), and other spectacular interactions (e.g., Neff et al. 2005; Duc \& Mirabel 1998). Even if we cannot confirm with the present data that it could have been pre-existing to the current interaction event, the Northern tail forms a physical connection between NGC 5713 and PGC 135857 and the Western tail has very similar velocities.

Concerning the evolution of the system, it is likely that the flow of accreted matter will enable star formation to continue for time-scales on the order of $1 \mathrm{Gyr}$ after the tidal feature formation (Hibbard \& Mihos 1995) and, as such, to have a strong impact on both the evolution and the dynamics of NGC 5719. The galaxy might eventually build a secondary stellar disc as massive as the main one, and evolve into an object similar to NGC 4550 (Rubin et al. 1992; Rix et al. 1992).

The alternative scenario involving only the accretion of already-formed stars is very unlikely due to the young age of the bright UV stars observed in the HI density peaks. However, we can not reject the accretion on retrograde orbit of a low-mass satellite that would partially contribute to the build up of the counter-rotating stellar population. The radial distribution of the counter-rotating component in NGC 5719 can be described as a disc with a central hole. This is qualitatively consistent with the results of the numerical simulations by Abadi et al. (2003), where most of the thick counter-rotating disc was assembled by stars from acquired and disrupted satellites. However, such a scenario needs the ad hoc assumption of a past accretion of a gasrich dwarf satellite. Two-dimensional optical spectroscopy that partially resolves the kinematics and maps the stellar age of the components should provide a definitive answer to this question.

\section{Conclusions}

The HI distribution and kinematics show an on-going major merger between the edge-on Sab spiral NGC 5719 and its Sbc spiral companion NGC 5713. Tidal features in the form of two bridges that loop around NGC 5719 connecting to NGC 5713, and two tails departing from NGC 5713 towards the West were detected. The total extent on the sky of the HI emission is about $27^{\prime}$, corresponding to $\sim 180 \mathrm{kpc}$ at the adopted distance. At the tip of the northern tail the low-mass satellite PGC 135857 was detected, which may represent a by-product of the interaction event.

The HI column density distribution within and outside the NGC 5719 disc is remarkably similar to the UV morphology, with a fairly precise correspondence with the location of the clumps in a young stellar population (200-500 Myr).

The neutral and ionised hydrogen in the disc of NGC 5719 are counter-rotating with respect to the main stellar disc. For the first time, a counter-rotating stellar disc which contains nearly $\sim 20 \%$ of the stars and which has the same radial extension as the main stellar disc has been detected in an interacting system.

The data support a scenario where neutral hydrogen was first accreted by NGC 5719 onto a retrograde orbit from the large HI reservoir available in the galaxy surroundings, and subsequently formed in situ into a counter-rotating stellar disc.

Acknowledgements. D.V. acknowledges supports by the European Commission through a Euro3D RTN on Integral Field Spectroscopy (No. HPRN-CT-200200305) and a Marie Curie ERG grant (No. MERG-CT-2005-021704). This research has made use of the Lyon-Meudon Extragalactic Database (HyperLeda), and the NASA/IPAC Extragalactic Database (NED) which is operated by the Jet Propulsion Laboratory, California Institute of Technology, under contract with the National Aeronautics and Space Administration. The Very Large Array (VLA) is operated by the National Radio Astronomy Observatory (NRAO). The NRAO is a facility of the National Science Foundation operated under cooperative agreement by Associated Universities, Inc.

\section{References}

Abadi, M. G., Navarro, J. F., Steinmetz, M., et al. 2003, ApJ, 597, 21 Athanassoula, E., Bosma, A., Guivarch, B., et al. 1996, in New light on galaxy evolution, ed. R. Bender, \& R. L. Davis, IAU Symp., 171, 339 Bender, R. 1990, A\&A, 229, 441

Bender, R., Saglia, R. P., \& Gerhard, O. E. 1994, MNRAS, 269, 785

Bertola, F., \& Corsini, E. M. 1999, in Galaxy Interactions at Low and High Redshift, ed. J. E. Barnes, \& D. B. Sanders, IAU Symp., 186, 149

Bertola, F., Cinzano, P., Corsini, E. M., et al. 1996, A\&A, 337, 80

Binney, J., \& Merrifield, M. 1998, Galactic astronomy (Princeton, NJ: Princeton University Press)

Carollo, C. M., Franx, M., Illingworth, G. D., et al. 1997, ApJ, 481, 710

Corsini, E. M., Pizzella, A., Funes, J. G., et al. 1998, A\&Ap, 337, 80

de Vaucouleurs, G., \& de Vaucouleurs, A. 1964, Reference Catalogue of Bright Galaxies (Austin: University of Texas Press) 
de Vaucouleurs, G, de Vaucouleurs, A. Corwin, J. R, et al. 1991, The Third Reference Catalogue of Bright Galaxies (New York: Springer-Verlag) (RC3)

Duc, P. A., \& Mirabel, I. F. 1998, A\&A, 333, 813

Emsellem, E., Cappellari, M., Peletier, R. F., et al. 2004, MNRAS, 352, 721

Evans, N. W., \& Collett, J. L. 1994, ApJ, 420, L67

Falcón-Barroso, J., Bacon, R., Bureau, M., et al. 2006, MNRAS, 369, 529

Garcia, A. M. 1993, A\&AS, 100, 47

García-Burillo, S., Sempere, M. J., Combes, F., et al. 2000, A\&A, 363, 869

Gerhard, O. E., Jeske, G., Saglia, R. P., et al. 1998, MNRAS, 295, 197

Guthrie, B. N. G. 1992, A\&AS, 93, 255

Hibbard, J. E., \& Mihos, J. C. 1995, AJ, 110, 140

Hibbard, J. E., Bianchi, L., Thilker, D. A., et al. 2005, ApJ, 619, 87

Jore, K. P., Broeils, A. H., \& Haynes, M. 1996, AJ, 112, 438

Kannappan, S. J., \& Fabricant, D. G. 2001, AJ, 121, 140

Kuijken, K., Fisher, D., \& Merrifield, M. R. 1996, MNRAS, 283, 543

Langston, G., \& Teuben, P. 2001, in Gas and Galaxy Evolution, ed. J. E. Hibbard, M. P. Rupen, \& J. H. van Gorkom, ASP Conf. Ser., 240, 862

McDermid, R. M., Bacon, R., \& Kuntschner, H. 2006, New Astron. Rev., 49, 521
Mendes de Oliveira, C., Cypriano, E. S., Sodre, L. Jr., et al. 2004, ApJ, 605, 17 Merrifield, M. R., \& Kuijken, K. 1994, ApJ, 432, 575

Merritt, D., \& de Zeeuw, P. T. 1983, ApJ, 267, 19

Mihos, J. C., \& Hernquist, L. E. 1994, ApJ, 427, 112

Mihos, J. C., \& Hernquist, L. E. 1996, ApJ, 464, 641

Morelli, L., Halliday, C., Corsini, E. M., et al. 2004, MNRAS, 354, 753

Morrissey, P., Schiminovich, D., Barlow, T. A., et al. 2005, ApJ, 619, 7

Mulchaey, J. S., Regan, M. W., \& Kundu, A. 1997, ApJS, 110, 299

Neff, S. G., Thilker, D. A., Seibert, M., et al. 2005, ApJ, 619, 91

Pizzella, A., Corsini, E. M., Vega Beltrán, J. C., \& Bertola, F. 2004, A\&A, 424, 447

Rhee, G., Valenzuela, O., Klypin, A., et al. 2004, ApJ, 617, 1059

Rix, H. W., Franx, M., Fisher, D., et al. 1992, ApJ, 400, 5

Roberts, M. S., \& Haynes, M. P. 1994, ARA\&A, 32, 115

Rubin, V. C., Graham, J. A., \& Kenney, J. D. P. 1992, ApJ, 394, L9

Schweizer, F. 2000, Phil. Trans. R. Soc. Lond., Ser. A, 358, 2063

Vergani, D., Dettmar, R.-J., van Driel, W., Gentile, G., \& Klein, U. 2006, A\&A, submitted

Wozniak, H., \& Pfenninger, D. 1997, A\&A, 317, 14 
D. Vergani et al.: NGC 5719/13: interacting spirals, Online Material p 1

\section{Online Material}


D. Vergani et al.: NGC 5719/13: interacting spirals, Online Material p 2

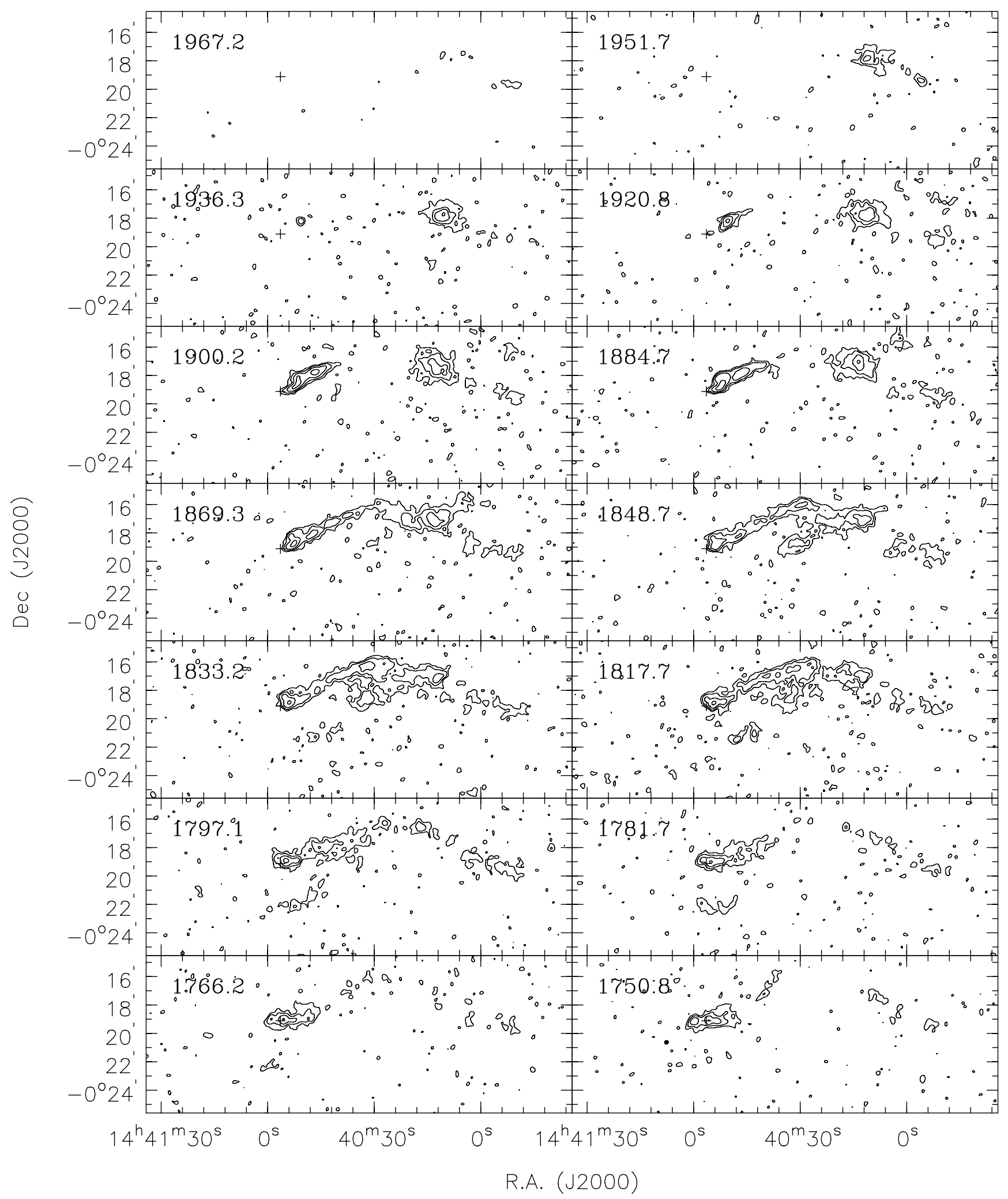

Fig. 4. Channel maps of the HI emission in the entire NGC 5719/13 system. Contour levels are at $-1.4,1.4(\sim 2.5 \sigma), 2.8,5.6$, and $11.2 \mathrm{mJy}^{-1}$ beam ${ }^{-1}$. The spatial resolution is $30^{\prime \prime} \times 30^{\prime \prime}$. The cross represents the optical centre of NGC 5719 . 
D. Vergani et al.: NGC 5719/13: interacting spirals, Online Material p 3

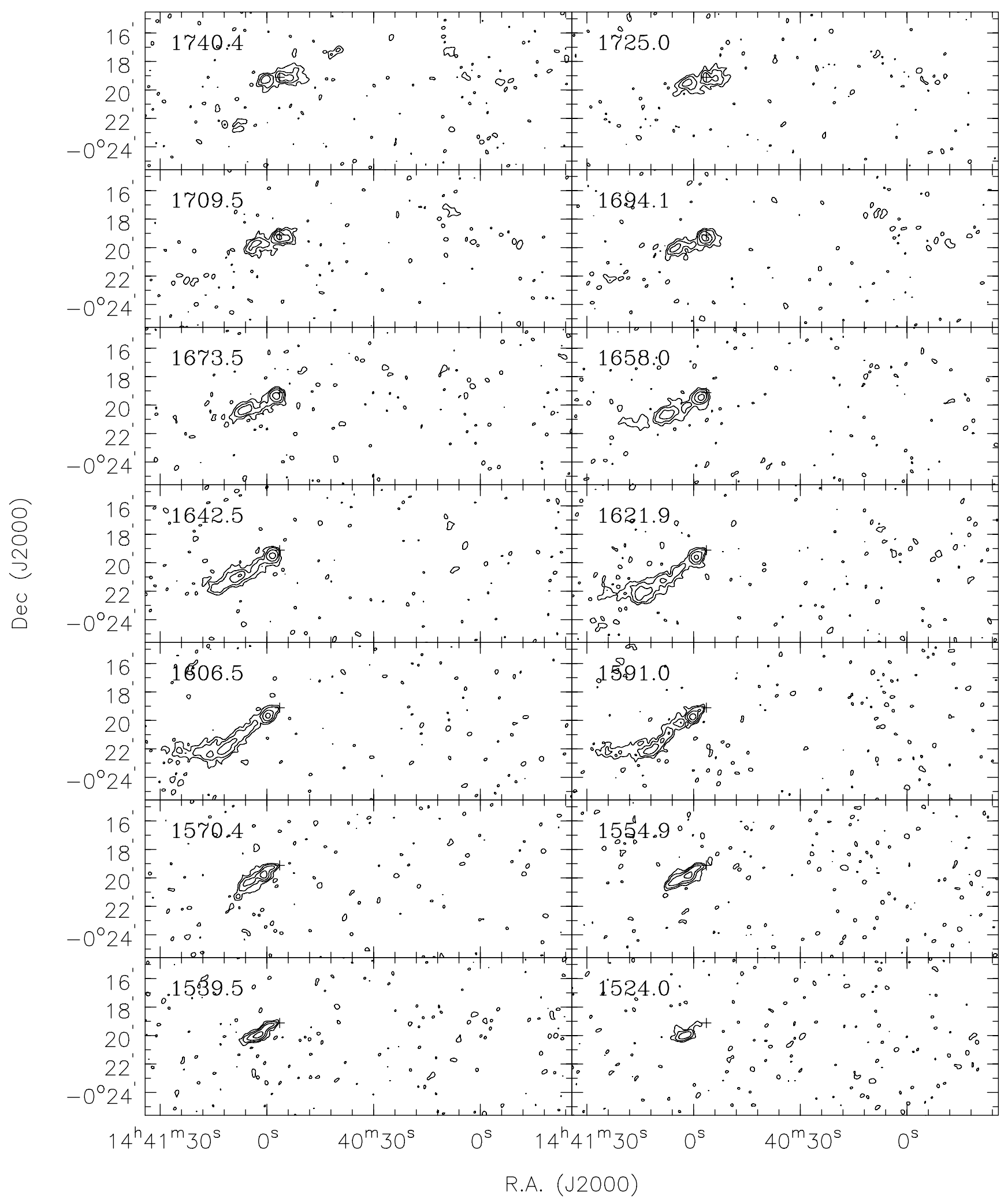

Fig. 4. continued. 